\title{
Fortbildung
}

\section{KINDER UND JUGENDLICHE AUS FAMILIEN MIT EINEM PSYCHISCH ERKRANKTEN ELTERNTEIL}

Kurt Albermann, Brigitte Müller

Kinder und Jugendliche mit einem psychisch erkrankten Elternteil sind mehr Belastungen ausgesetzt und haben ein deutlich erhöhtes Risiko, selbst psychische Störungen zu entwickeln. Weltweit sind ca. 15 bis 23\% aller Kinder betroffen, auf die Schweiz übertragen entspricht dies ungefähr 300'000 Kinder und Jugendliche unter 18 Jahren $^{1,2)}$. In diesem Beitrag werden die Auswirkungen einer elterlichen psychischen Erkrankung auf die Gesundheit der betroffenen Kinder und Jugendlichen beschrieben. Zudem wird aufgezeigt, wie sich diese erkennen lassen und wie Kinder und Familien ressourcenorientiert unterstützt werden können.

\section{Psychische Gesundheit}

Psychische Gesundheit ist nicht ein Zustand, sondern entsteht durch vielfältige und dynamische Wechselwirkung zwischen Ressourcen und Belastungen eines Individuums. Sowohl die Intensität und Häufigkeit von Belastungen (Stressoren), die individuelle Vulnerabilität, als auch die verfügbaren Ressourcen (Stärken, Schutzfaktoren) spielen dabei eine Rolle, um Stresssituationen zu bewältigen ${ }^{3}$. Im Kontext der psychischen Gesundheit Erwachsener wird diese unterdessen auch als «well-being» mit verschiedenen Dimensionen bezeichnet, meint also emotionales, psychisches und soziales Wohlbefinden ${ }^{4-6)}$. Im günstigsten Fall umfassen diese positiven Emotionen: Glück, Lebenszufriedenheit, das Erkennen und Ausschöpfen eigener Fähigkeiten und stabile, förderliche Beziehungen mit anderen sowie ein Beitrag zur Gemeinschaft. Kinder und Jugendliche sind im Hinblick auf ihr Wohlbefinden - abhängig von ihrem Entwicklungsalter - sehr auf feinfühlige, liebevolle und stabile Bezugs- und Betreuungspersonen, auf Geborgenheit und förderliche Umgebungsbedingungen angewiesen. Diese ermöglichen ihnen, altersentsprechende Lebenserfahrungen zu machen, daher kommt in diesem gewissermassen «abhängigen», Lebensalter dem psychosozialen Kontext eine besondere Bedeutung zu. Ein integratives Verständnis psychischer Gesundheit umfasst in einem erweiterten bio-psycho-sozialen Modell ${ }^{7}$ zudem auch genetische, epigenetische und individuellen Faktoren ${ }^{7,8)}$.

\section{Psychische Störungen}

Psychische Störungen beeinträchtigen die Wahrnehmung, das Denken und die Emotionen. Sie wirken sich auf das Erleben und Verhalten der Betroffenen aus ${ }^{9}$. Betroffene Personen leiden häufig selbst darunter, allerdings fehlt Kindern und Jugendlichen, abhängig vom Entwicklungsalter, unter Umständen (noch) die Fähigkeit, ihre Wahrnehmung und ihr Empfinden so zum Ausdruck zu bringen, dass sie von den Eltern resp. von ihrem Umfeld richtig "gelesen und interpretiert» werden. Auch erfahrenen Fachpersonen fällt dies im Übrigen nicht immer ganz leicht. Eigentliches Leiden und Behandlungsbedarf ergibt sich in der Regel dann, wenn das Kind, die Eltern oder die Schule dies berichten und eine Abweichung des gewohnten Verhaltens feststellen resp. die Partizipation im Alltag und die Leistungsfähigkeit z.B. in der Schule eingeschränkt ist. Die Zeit bis zum Aufsuchen einer Fachperson ist häufig für alle Beteiligten sehr belastend, die Inanspruchnahme von Abklärung, Beratung und Therapie aus verschiedenen Gründen allerdings viel zu häufig suboptimal.

\section{Kasuistik}

Somit ist es gut vorstellbar, dass beispielsweise Frau R. mit einer floriden schizophrenen Erkrankung nur eingeschränkt in der Lage ist, die Bedürfnisse ihrer beiden Kinder wahrzunehmen. Die 10jährige Annina und ihr drei Jahre jüngerer Bruder Ben können die langsam eintretenden Veränderungen bei ihrer Mutter nicht einordnen, sind verunsichert und verwirrt, fühlen sich schuldig. Sie schämen sich für ihre Mutter, wenn sie zusammen beim Einkauf sind, weil die Mutter wirr redet, sich von den an der Decke angebrachten Kameras fürchtet und deshalb mit aufgespanntem Regenschirm durch den Laden geht. Nachts wurde von den Nachbarn bereits einmal die Polizei herbeigerufen und die Mutter in Handschellen schreiend und randalierend zur stationären Therapie in die Psychiatrie eingeliefert. Die Kinder haben Angst und beginnen sich vor ihr zu fürchten. Sie verlieren zunehmend den Boden unter den Füssen. Der Vater ist verzweifelt, weiss sich kaum zu helfen. Er beginnt zu trinken und ist den Kindern kaum eine Stütze, da er in leitender Position berufstätig ist und über wenig zeitliche und persönliche Ressourcen verfügt. Ihm ist es peinlich, sich bei seinem Arbeitgeber zu outen und um Verständnis und Entlastung zu bitten. Im weiteren Verlauf beginnt Ben in der ersten Klasse wieder einzunässen und prügelt sich mit anderen Kindern. Der Vater beschliesst, sich von seiner Frau zu trennen, Annina kümmert sich um Ben und den Haushalt, vernachlässigt ihre Hobbies und zieht sich zunehmend auch von ihren Freundinnen zurück. Nach mehreren Monaten erreicht den Vater eine Gesprächseinladung der Schulbehörde: Anninas Notendurchschnitt habe

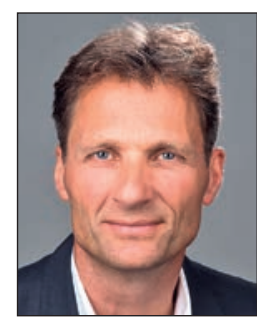

Kurt Albermann

https://doi.org/ 10.35190/d2021.4.5
Korrespondenz: kurt.albermann@ksw.ch 


\section{Fortbildung}

sich um mehr als eine Note verschlechtert, sie sei unkonzentriert und häufig abgelenkt. (Abbildung 1)

\section{Prävalenz}

Gemäss verschiedenen Studien ist die Wahrscheinlichkeit für Kinder, eine psychische Störung zu entwickeln, um den Faktor 3 bis 7 erhöht, wenn sie in einer Familie aufwachsen, in der ein Elternteil psychisch erkrankt ist. Kinder, die aufgrund einer schweren psychischen Belastung oder Störung beraten oder behandelt werden, stammen häufig aus Familien, in denen auch die Eltern von psychischen Belastungen oder Erkrankungen betroffen sind. Im Vergleich zu Kindern ohne psychische Erkrankung leben psychisch kranke Kinder deutlich häufiger in Familien mit schwieriger Familiendynamik (11.7 vs. 28.3\%) ${ }^{10)}$. Das Erkrankungsrisiko eines Kindes mit einem schizophrenen Elternteil erhöht sich vom Durchschnitt der Gesamtbevölkerung (1 Prozent) auf ca. 13 Prozent. Bei einer schizophrenen Erkrankung beider Eltern erhöht sich das lebenslange Risiko für ihre Kinder, selbst eine solche Störung zu entwickeln, sogar auf etwa $40 \%{ }^{111}$. Das Risiko für eine Depression ist bei elterlicher depressiver Erkrankung etwa um das zwei- bis sechsfache erhöht. Etwa 60\% der Kinder von Eltern mit einer Depression entwickeln im Verlauf der Kindheit und Jugend eine psychische Störung ${ }^{12)}$. Eine repräsenta- tive Erhebung für die Schweiz aus dem Jahr 2017 zeigte, dass 7,9\% der befragten 3991 Kinder und Jugendlichen zwischen 10 und 15 Jahren regelmässig und massgeblich Verantwortung für die Betreuung und Pflege einer nahestehenden Person übernehmen 13). International werden Minderjährige in vergleichbarer Lebenssituation als Young Carers bezeichnet. Sie bilden je nach Alter und familiärer Konstellation bei elterlicher psychischer Erkrankung eine Schnittmenge mit weiter oben genannten Gruppen. In der Schweiz leisten sie häufig emotionale, betreuerische und pflegerische Unterstützung sowie Hilfe im Haushalt.

\section{Auswirkungen der COVID-19 Pandemie auf die psychische Gesundheit}

Die aussergewöhnlichen Belastungen im Zusammenhang mit der Covid-19-Pandemie haben in zahlreichen Familien die Balance zwischen Ressourcen und Stressoren aus dem Gleichgewicht gebracht: finanzielle Sorgen, Arbeitslosigkeit sowie Doppelbelastung durch Kinderbetreuung und Homeoffice führten zu mehr Konflikten, erhöhten den familiären Druck und wurden für manche sogar existenzbedrohend. Bewährte Copingstrategien fielen durch die Beschränkung der sozialen Austauschmöglichkeiten, die vorübergehende Schliessung von Vereinen, Kultur- und Sporteinrich-

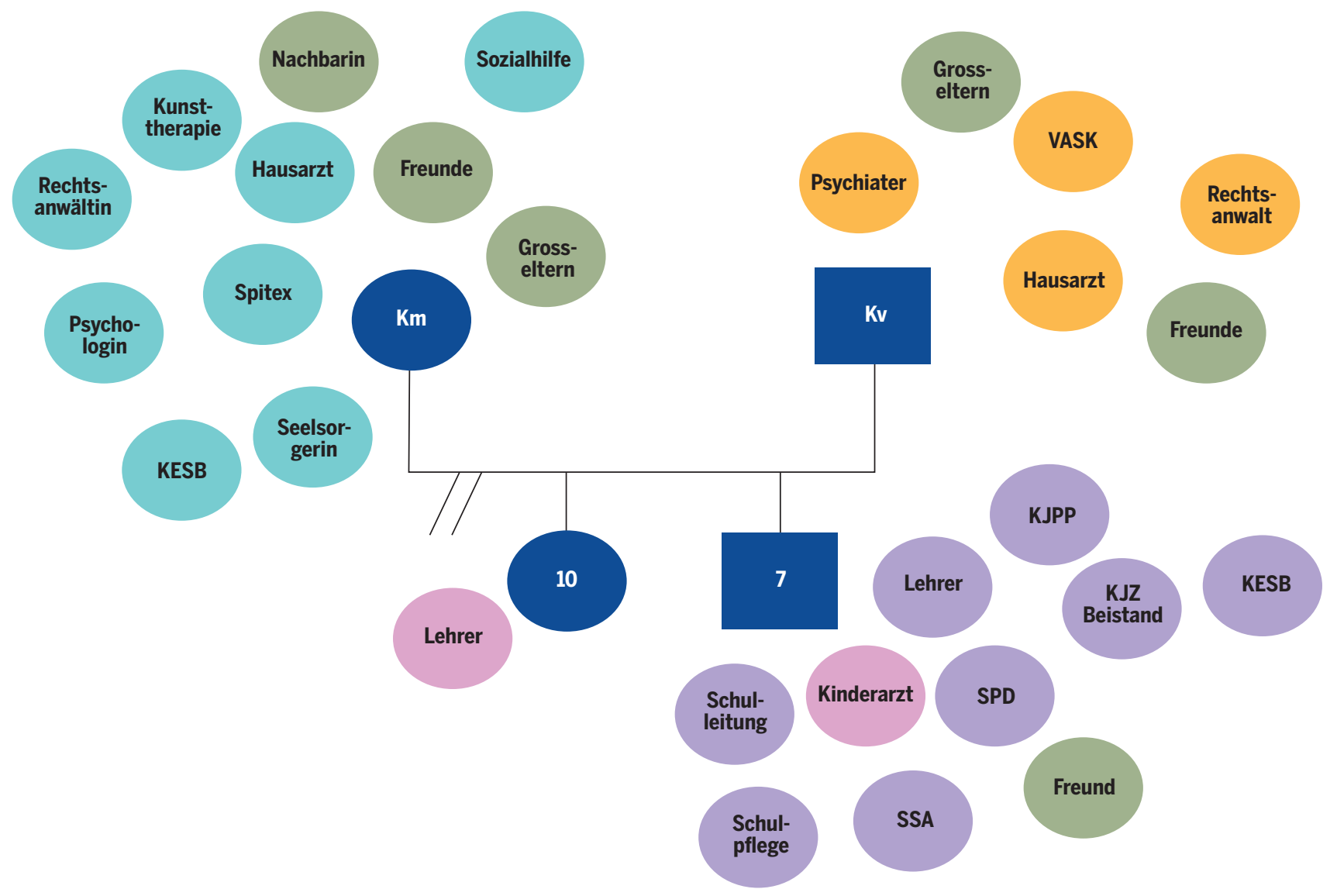

Abb. 1. Darstellung des Familiensystems von Familie R. mit ihren beiden Kindern Annina (10jährig) und Ben (7jährig), die Mutter (Km) ist an Schizophrenie erkrankt, der Vater (Kv) hat sich deshalb von ihr getrennt. Die Familie benötigt ein umfangreiches Versorgungsnetz, die Km befindet sich in stationärer Behandlung. KESB (Kindes- und Erwachsenenschutzbehörde), SPD (Schulpsycholiogischer Dienst), SSA (Schulsozialarbeit), KJZ (Kinder- und Jugendhilfezentrum), KJPP (Kinder- und Jugendpsychiatrie und Psychotherapie, VASK (Vereinigung der Angehörigen von psychisch Kranken) 


\section{Fortbildung}

tungen und auch der Schulen weg oder wurden stark reduziert. Die Nutzungsdaten der «Dargebotenen Hand», von Pro Juventute sowie die Rückmeldungen von Fachpersonen der Kinder- und Jugendpsychiatrie zeigen, dass Beratungsanfragen im Zusammenhang mit Suizidgedanken im Kontext der Pandemie zugenommen haben ${ }^{14)}$. Eine in England durchgeführte Umfrage zeigte, dass Kinder und Jugendliche mit Aufmerksamkeitsdefizit-/Hyperaktivitätsstörung (AHDS) und Autismus-Spektrum-Störung (ASS) sowie mit besonderen pädagogischen Bedürfnissen oder aus Familien mit geringem Einkommen sich von den Einschränkungen des Lockdowns schlechter erholten und weiterhin Symptome psychischer Belastung aufwie$\operatorname{sen}^{15)}$. Eine anonyme Online-Umfrage der Universitäten Zürich und Bern zu den Auswirkungen der Corona-Pandemie zeigte bei Vorschulkindern gemäss Angaben der Eltern mehr trotziges und aggressives Verhalten, bei den 11-19-Jährigen mehr emotionale Probleme wie Ängste und Depressionen. Bei den 1-10-Jährigen hingen psychische Probleme der Eltern mit allen Formen von psychischen Problemen der Kinder zusammen. Während jüngere Kinder sehr von den elterlichen Copingstrategien, Ressourcen und deren sozioökonomischer Situation abhängig waren, kamen Jugendliche durch ihre unabhängigeren sozialen Kontakte mit den Peers oftmals besser zurecht ${ }^{16)}$. Weitere Erhebungen zeigten heterogene Reaktionen auf die Pandemie-Situation bei Jugendlichen: Einige reagierten mit erhöhtem Stress und Konzentrationsstörungen, bei anderen führten der transiente Lockdown und die Schulschliessungen zu einer Stressreduktion, weniger psychosozialem Druck durch die Peers, zu erhöhtem Wohlbefinden und mehr freier Zeit ${ }^{14,17)}$.

\section{Auswirkungen auf die Familie}

Familien mit einem psychisch erkrankten Elternteil stellen eine sehr heterogene Gruppe dar. Für die Familie und speziell für die Kinder ist es von grosser Bedeutung, welcher Elternteil von der Erkrankung betroffen ist, wie lang sie besteht und auf welche Weise sie sich auf die familiären Beziehungen, die Kommunikation und nicht zuletzt auf die Alltagsorganisation auswirkt. Deutlich häufiger als alleinerziehende Väter übernehmen alleinerziehende Mütter trotz psychischer Erkrankung Kinderbetreuungsaufgaben. Nicht selten müssen sie zudem den Lebensunterhalt für die Familie verdienen ${ }^{18}$. Ein Klinikaufenthalt einer Mutter mit jüngeren Kindern bringt gravierende Veränderungen mit sich, insbesondere, wenn die Mutter alleinerziehend ist. Das familiäre Netzwerk ist, wie oben dargestellt, extrem gefordert. Nicht immer können Grosseltern, enge Freunde oder Nachbarn einspringen. Freiwillige oder professionelle Entlastungsdienste und die Angebote der Kindes- und Jugendhilfe bieten häufig keine lückenlose Tagesbetreuung an. Manchmal ist deshalb eine vorübergehende Unterbringung der Kinder in einer anderen Familie oder in einer Institution unumgänglich.

\section{Belastungen von Kindern und Jugendlichen}

Die Auswirkungen einer elterlichen psychischen Erkrankung oder Suchterkrankung sind unspezifisch. Im Rahmen von Vorsorgeuntersuchungen ist es möglich,
Kinder, Jugendliche und Eltern auf besondere Belastungen anzusprechen. Es ist hilfreich, auf der Basis einer vertrauensvollen Beziehung konkret nachzufragen, wie der Alltag läuft, resp. was sich Kinder und Eltern konkret anders wünschen würden, wer zu Hause welche Aufgaben übernimmt, ob und wie das jeweils funktioniert. Nach einer Geburt lohnt sich der Austausch mit der Mütter-/Väterberatung resp. mit den Hebammen. Sie erleben Kinder und Familien direkt im familiären Umfeld und können auf Belastungen und Unterstützungsbedarf hinweisen. Die Häufigkeit einer postpartalen Depression beträgt für Mütter ca. 15\%, für Väter ca. 10\%. Es ist wichtig, auch die Väter in die Beratung einzubeziehen und zu gemeinsamen Gesprächen einzuladen. Kinder von Eltern mit Persönlichkeitsstörungen und Abhängigkeitserkrankungen sind offenbar am meisten gefährdet. Insbesondere Kinder von Müttern mit einer Borderline-Persönlichkeitsstörung leiden unter den Auswirkungen der mütterlichen Erkrankung. Von einer elterlichen psychischen Erkrankung betroffene Kinder zeigen auch häufiger Verhaltensauffälligkeiten und Störungen im sozialen, emotionalen und kognitiven Bereich. Young Carers können auf verschiedene Weise unterstützt werden, z.B. über direkte Aufklärung und Informationen, praktische Alltagshilfen und Beratungen oder indirekt über Leistungen an die zu unterstützende Person bzw. das gesamte Familiensystem ${ }^{8)}$. Zudem äussern Young Carers in der Schweiz den Wunsch nach mehr Akzeptanz durch Fachpersonen ${ }^{19}$. Wertschätzung und Anerkennung für die geleistete Unterstützung wirken sich stressmindernd auf diese Kinder und Jugendlichen aus ${ }^{20)}$.

\section{Ressourcen im familiären Netzwerk}

Bei einer psychischen Erkrankung eines Elternteils entstehen viele Fragen und Unsicherheit in Bezug auf die Organisation des Familienalltags. Manche Familien können auf ein funktionierendes Netzwerk im privaten oder öffentlichen Bereich zurückgreifen und erhalten von dort eine ihrer Lebenslage und derzeitigen Lebenssituation entsprechende Unterstützung. Das hängt mitunter sehr stark vom Erkrankungsstadium ab. Bei einer Erstmanifestation sind oft noch innerfamiliäre Ressourcen vorhanden. Sobald sich ein chronischer Krankheitsprozess etabliert, findet sich häufig ein zunehmend erschöpftes familiäres Unterstützungssystem. Bei Alleinerziehenden fehlt oft die Einbettung der Familie in ein Verwandtschafts-, Freundes- und Nachbarsystem. Dieses fehlt dann auch bei Erkrankung oder elterlicher Trennung, Umzug und wirtschaftlichen Problemen. Bei Chronifizierung einer psychischen Erkrankung gewinnen Netzwerke aus dem öffentlichen Bereich mit zunehmender Komplexität der Probleme auf der sozialen, wirtschaftlichen und familiären Lebensachse an Bedeutung. Wenn keine oder eine unzureichende Vernetzung besteht, sich der betroffene Elternteil oder wie im obigen Beispiel die ganze Familie R. sich aus Scham, auch krankheitsbedingtem Misstrauen oder wegen negativer Erfahrungen zurückzieht, resultiert nicht selten eine Negativspirale zunehmender sozialer Isolation. 


\section{Fortbildung}

Wer spricht mit den Kindern und Jugendlichen?

Partner, Angehörige und Kinder stehen diesen Prozessen häufig weitgehend hilflos gegenüber. Doch wollen und müssen Kinder und Jugendliche ihrem Alter und ihrem Wissensstand entsprechend informiert und in das häufig komplexe Management der elterlichen psychischen Erkrankung einbezogen werden. Jüngere Kinder wünschen sich Informationen durch den betroffenen Elternteil, Jugendliche hingegen bevorzugen den Einbezug und Informationen durch Fachpersonen in Praxis oder Klinik. Sie sind allerdings mitunter recht kritisch und befürchten, keine objektiven Informationen zu erhalten ${ }^{21}$. Psychisch erkrankte Eltern, Familien und ihre Kinder erhalten jedoch zumeist weder während des Spitalaufenthaltes, noch nach Entlassung entsprechende Informationen. Die beratenden und behandelnden Ärzte und Fachpersonen im Versorgungssystem für Erwachsene fühlen sich in erster Linie ihren Klienten verpflichtet, weniger deren Kindern, die sie häufig weder kennen noch in die Behandlung einbeziehen (Abbildung 2) ${ }^{22,23)}$. Daher ist es notwendig, mit Eltern über die Bedürfnisse ihrer Kinder zu sprechen, Ängste der Eltern zu thematisieren und gemeinsam Gespräche über die elterliche psychische Erkrankung zu führen und auf Fragen der Kinder einzugehen (Tabelle 1).

\section{Diagnostik und Material}

Hierbei kann es hilfreich sein, altersadäquates Aufklärungsmaterial, also themenspezifische Kinderbücher, Handpuppen, Plüschtiere, Broschüren oder auch Filmmaterial einzusetzen. Der Einsatz von Fragebögen kann orientierend Auskunft über kindliche Belastun- gen geben. Für die weitergehende Diagnostik sind Psycholog*innen oder die Kinder- und Jugendpsychiatrie beizuziehen. Mit dem Strengths and Difficulties Questionnaire (SDQ) lassen sich beispielsweise Verhaltensstärken und -auffälligkeiten bei Kindern und Jugendlichen im Alter von 4 bis 16 Jahren erfassen. Der SDQ zeigte für die Eltern-, Lehrer- und Selbst-Versionen für Jugendliche eine hohe Korrelation mit längeren etablierten Fragebögen ${ }^{24,25)}$. Das Ausfüllen benötigt ca. fünf Minuten. In der pädiatrischen Praxis besteht im Rahmen der Vorsorgeuntersuchungen Gelegenheit, Eltern, in der Regel Mütter, auf individuelle und familiäre Belastungen, Schutz- und Risikofaktoren anzusprechen und auf professionelle Hilfen hinzuweisen. Leider gibt kein Patentrezept - für jede Familie müssen die für sie geeigneten Unterstützungsangebote gefunden werden.

\section{Unterstützungsangebote}

Mittlerweile gibt es in der ganzen Schweiz diverse Beratungs- Behandlungs- und Unterstützungsangebote für Kinder, Jugendliche und Familien mit psychisch und suchterkrankten Eltern. An einigen psychiatrischen Einrichtungen für Erwachsene (z.B. Königsfelden) wurden Sprechstunden für Angehörige und Kinder eingerichtet, die eng mit der Kinder- und Jugendpsychiatrie zusammenarbeiten (z.B. Chur, Luzern). Zudem existieren Elterngruppen (z.B. in Bern, Zürich, Winterthur) oder integrierte Eltern-Kind-Angebote für Mütter mit Säuglingen und Kleinkindern (z.B. Affoltern) und für Mütter/Väter mit Vorschulkindern bis max. 5 Jahre (z.B. Münsterlingen) ${ }^{26)}$. Der Kanton Waadt hat mit ZigZag Plus ein Beratungs- und Unterstützungsangebot für Kinder von psychisch erkrankten

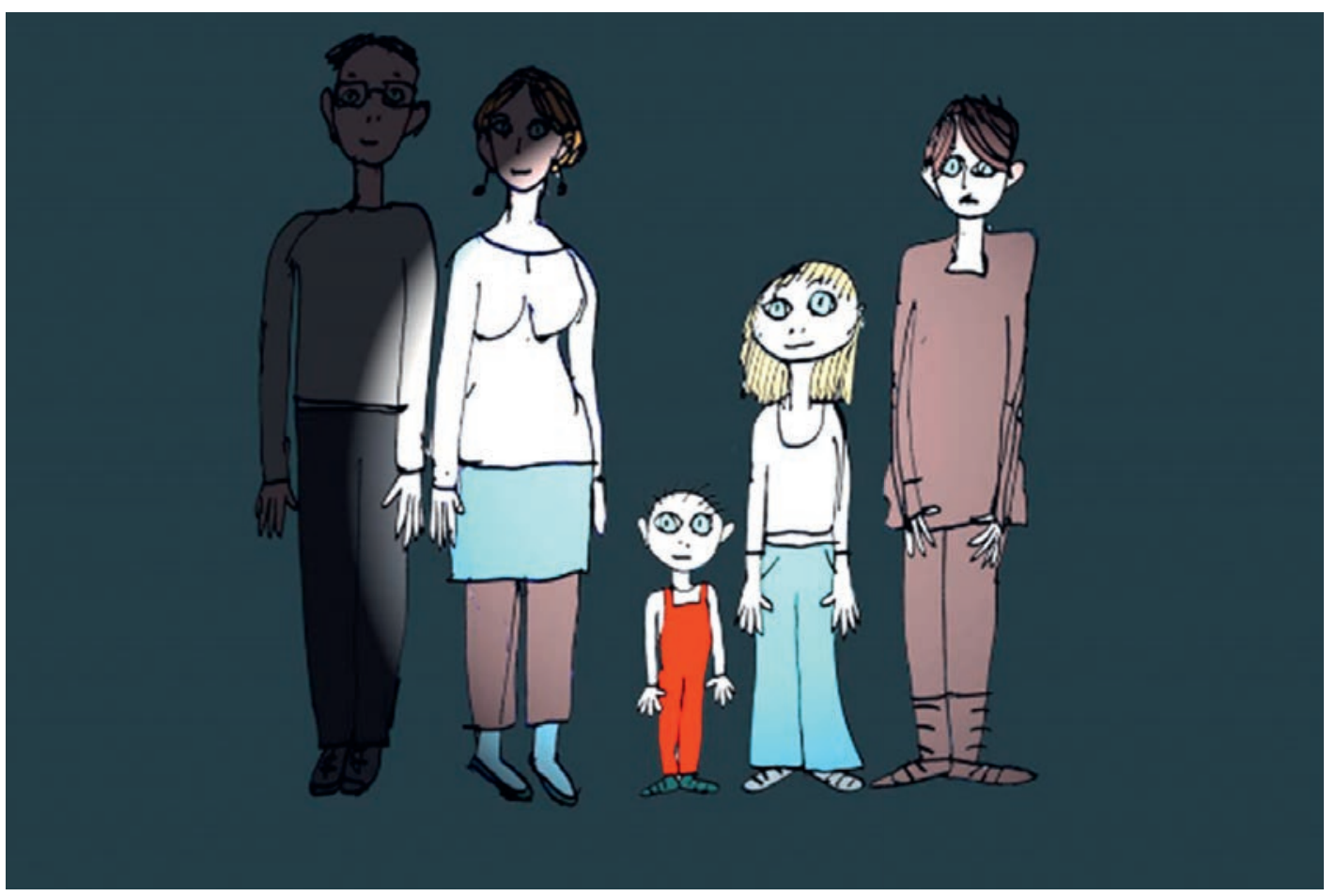

Abbildung 2. Im Versorgungssystem für Erwachsene liegt der Fokus der behandelnden Person beim einzelnen Patienten, also beim jeweils behandelten Elternteil (dunkel dargestellt). Manchmal wird der gesunde Elternteil einbezogen, selten bis nie die Kinder. Gerade die Kinder benötigen aber Aufklärung, Einbezug und Information (deshalb hier im Fokus und hell dargestellt). www.liliancaprez.ch 


\section{Fortbildung}

\begin{tabular}{l|l}
\hline $\begin{array}{l}\text { Verstehen, was passiert ist } \\
\text { (Krankheitsursachen und Verlauf) }\end{array}$ & $\begin{array}{l}\text { Was ist los mit Mutter oder Vater? } \\
\text { Warum sind sie krank geworden? } \\
\text { Bin ich schuld daran? Habe ich etwas falsch gemacht? } \\
\text { Wird es wieder gut werden? Wie lange dauert die } \\
\text { Krankheit? }\end{array}$ \\
\hline $\begin{array}{l}\text { Wie soll ich mich verhalten? } \\
\text { (Umgang im familiären Alltag) }\end{array}$ & $\begin{array}{l}\text { Was muss ich beachten. Was soll ich anders machen? } \\
\text { Wie soll ich auf das veränderte Verhalten reagieren? } \\
\text { Was mache ich, wenn es zu Hause schwierig wird? }\end{array}$ \\
\hline $\begin{array}{l}\text { Wie geht es mit mir weiter? } \\
\text { (Veränderungen im Familienleben) }\end{array}$ & $\begin{array}{l}\text { Wer kümmert sich jetzt um mich? } \\
\text { Wem kann ich vertrauen? Wer ist für mich da? } \\
\text { Darf ich darüber sprechen? Mit wem? }\end{array}$ \\
\hline $\begin{array}{l}\text { Was bedeutet eine psychische Krankheit? } \\
\text { (Information über psychische Störungen) }\end{array}$ & $\begin{array}{l}\text { Was heisst eigentlich «psychisch krank»? } \\
\text { Wird die Mutter / der Vater wieder gesund? } \\
\text { Was kann ich tun? }\end{array}$ \\
\hline $\begin{array}{l}\text { Gibt es Medikamente? Was passiert in der Therapie? } \\
\text { Kann ich auch krank werden? }\end{array}$ \\
\hline $\begin{array}{l}\text { Was mache ich wenn...? } \\
\text { (Notfallplan 24/7) }\end{array}$ & $\begin{array}{l}\text { Wer hilft mir? Wen kann ich wann anrufen? } \\
\text { Wo bin ich sicher? Wo kann ich wann hingehen? } \\
\text { Wer hilft dann der Mutter / dem Vater? } \\
\text { Soll ich das 144 oder 117 anrufen? }\end{array}$ \\
\hline
\end{tabular}

Tabelle 1. Informationsbedürfnis von Kindern und Jugendlichen (in Anlehnung an Lenz et al. 201621)

und suchtkrankten Eltern aufgebaut, das auch in den Kantonen Fribourg, Neuchâtel und im Wallis etabliert werden soll. Die Stiftung As'trame bietet in der ganzen Romandie unter anderem Unterstützung für Kinder psychisch erkrankter Eltern an. Einige Städte/Kantone bieten Patenschaften (z.B. Basel, Winterthur) oder eine SOS-Kinderbetreuung für betroffene Familien (Region Winterthur), sowie Mal- oder Gesprächsgruppen und Einzelberatung / -therapie für Kinder psychisch oder suchterkrankter Eltern an (z.B. Zebra, Windlicht in Winterthur, Kinderprojekt Barca). Der Verein «Postpartale Depression Schweiz» klärt über diese Krankheit auf und setzt sich dafür ein, dass die Betroffenen rasch die richtige Hilfe finden.

Von einer psychischen Erkrankung eines Elternteils betroffene Familien unterscheiden sich auch bezüglich dem, was sie brauchen («needs»). Die subjektiven Bedürfnisse und von Fachpersonen wahrgenommene Bedarfslagen können dabei erheblich voneinander abweichen. Aufsuchende Unterstützungs- und Beratungsangebote oder Hometreatment können den Bedürfnissen betroffener Familien entgegenkommen (z.B. HotA/Aargau, MST-CAN/Basel, Thurgau). Das REPR (Relais Enfants Parents Romands, anciennement Carrefour Prison) begleitet Kinder inhaftierter Personen in der Romandie im Besuchszimmer sowie vor und nach den Besuchen und unterstützt die Angehörigen. Careum setzt sich für die Rechte und angemessene Unterstützung von «Young Carers» ein.

Das «Institut Kinderseele Schweiz» (iks) betreibt als Schweizerische Stiftung zur Förderung der psychischen Gesundheit von Kindern und Jugendlichen eine umfassende (deutschsprachige) Website mit wertvollen Tipps, Materialien und Links zum Thema. Iks hat zahlreiche Kurzfilme für Jugendliche, Eltern und Fachpersonen produziert, die über diverse psychische Er- krankungen informieren sowie zum Umgang mit einer elterlichen psychischen oder Suchterkrankung.

Gemeinsam mit dem Kanton Thurgau hat iks ein online-basiertes Weiterbildungstool für Mitarbeitende an psychiatrischen Kliniken und Fachstellen entwickelt, und mit dem Kanton St. Gallen eine Kontaktstellenplattform mit Adressen und Unterstützungsangeboten für die Deutschschweiz. Nicht zuletzt bietet iks mit Partnerinstitutionen per Online-Chat, telefonisch oder vor Ort in Winterthur und Stäfa Beratung und Unterstützung für Kinder und Jugendliche, Eltern, weitere Angehörige und Fachpersonen an.

\section{Akzeptanz und familiäre Autonomie}

Nicht jede Familie benötigt dieselben Hilfsangebote die familiäre Autonomie ist in jedem Fall zu respektieren und es sollte gemeinsam besprochen werden, in welchen Lebensbereichen Unterstützung benötigt wird, die den familiären Bedürfnissen bestmöglich entspricht. Manche Eltern sind gut über Unterstützungsmöglichkeiten informiert. Allerdings ist für sie mit dem Kontakt zum Hilfssystem nicht selten die Angst verbunden, dass ihnen die Kinder entzogen und allenfalls fremduntergebracht werden ${ }^{27}$. Betroffene Eltern lassen sich im Gespräch häufig dadurch gewinnen, dass innen in dieser Hinsicht Verständnis entgegengebracht wird. Es ist hilfreich anzuerkennen, dass auch psychisch erkrankte Eltern im Grunde das «Beste» für ihr Kind möchten, dass sie wünschen, dass ihr Kind seine Fähigkeiten entfalten und sich zu einem selbstbestimmten, glücklichen Menschen entwickeln kann. Wenn Eltern wegen einer psychischen oder einer anderen schweren Erkrankung nicht mehr alle Erziehungsaufgaben wahrnehmen können, kann es notwendig werden, diese Verantwortung, vorübergehend oder bei Bedarf auch längerfristig, teilweise oder vollständig, anderen Personen zu übertragen. 
Deshalb wurden im Beispiel der Familie R. Beistandschaften für die Mutter und für Ben sowie weitere Unterstützung etabliert. Ben und später auch Annina erhielten psychotherapeutische Unterstützung, um die erfahrene Traumatisierung zu bearbeiten und mit den fortbestehenden elterlichen und psychosozialen Belastungen einen angemessenen Umgang zu finden. Auch in der Schule fanden Gespräche statt, die Lehrpersonen mit den notwendigen Informationen versorgten, um beide Kinder bestmöglich zu unterstützen $\left(v g l\right.$. dazu $\left.{ }^{28)}\right)$. Der Vater reduzierte sein Arbeitspensum, die Mutter lebte bis auf weiteres in einer betreuten Wohngruppe. Durch spezifische Entlastungsund Unterstützungsmassnahmen lassen sich persönliches Leid, Folgeerkrankungen und langfristig die volkwirtschaftlichen Kosten häufig reduzieren, die Lebensqualität der Kinder und Eltern hingegen zumeist deutlich erhöhen.

\section{Kinder benötigen Geborgenheit, verlässliche und förderliche Beziehungserfahrungen und ein sicheres, stabiles Umfeld.}

\section{Gelingende Unterstützung}

Eine gelingende Unterstützung orientiert sich immer am Erkrankungsstadium, an der Ausprägung der Erkrankung, der dazugehörenden Symptomatik sowie am aktuellen Informationsbedarf und den Copingstrategien der Familie und der Kinder. Betroffene Familien sollten die Möglichkeit haben, sich im Netzwerk an Fachpersonen ihres Vertrauens zu wenden (Ansatz des «no wrong door»). In der pädiatrischen oder hausärztlichen Praxis besteht, im Rahmen der Vorsorgeuntersuchungen, Gelegenheit, Eltern, in der Regel Mütter, auf individuelle und familiäre Belastungen, Schutz- und Risikofaktoren anzusprechen. Kinder und Jugendliche können auch direkt auf Belastungssituationen angesprochen werden. Allerdings werden sie aus Loyalität der Familie gegenüber und aus Angst vor unvorhergesehenen Konsequenzen in den wenigsten Fällen eine Antwort geben, welche die Familie belasten könnte. Ebenso wenig ist im Kindergarten oder in der Schule zu erwarten, dass Kinder und Jugendliche von sich aus auf ihre Belastungen aufmerksam machen.

Daher ist gemeinsam mit Eltern und Schulen und in der Öffentlichkeit ein geeigneter Diskurs zu führen, um der Tabuisierung psychischer Erkrankungen zu begegnen. Erst dadurch kann es möglich werden, an geeigneten Fachstellen und mit Personen, denen Kinder vertrauen, frühzeitig auf psychische Erkrankungen im Allgemeinen und auf elterliche psychische, körperliche und Suchterkrankungen im Besonderen hinzuweisen $^{29)}$.

\section{Kinderschutzmassnahmen}

Kinder werden nur im äussersten Notfall ausserhalb der Familie untergebracht. Familien stehen verschiedene niederschwellige Angebote offen, die sie in Erziehungsfragen und bei Herausforderungen im Fami- lienalltag unterstützen, wenn sie Belastungen nicht mehr selbst bewältigen können ${ }^{8)}$. Zudem sind in den meisten grösseren Gemeinden Beratungsangebote für Kinder und Jugendliche, Erziehungs- und Familienberatungsstellen, die Schulsozialarbeit oder «Hilfen zur Erziehung» bei besonderem Förder- oder Betreuungsbedarf verfügbar. In sehr problematischen Familiensituationen kann auch eine Sozialpädagogische Familienbegleitung vor Ort zum Einsatz kommen. Es ist jeweils individuell und verlaufsbezogen zu entscheiden, welche Unterstützungsmassnahmen geeignet sind, psychisch erkrankte Eltern und Familien in der Ausübung ihrer Erziehungsverantwortung zu unterstüt$z^{30}{ }^{30}$. Betroffene Familien wollen in den Entscheidungsprozess betreffend möglicher Förder- und Hilfsmassnahmen aktiv einbezogen und daran beteiligt werden, gemäss dem Motto «Don't talk about us without us». Falls eine unmittelbare und schwere Gefährdung eines Kindes vorliegt oder zu erwarten ist, sind sofortige Massnahmen zum Schutz des Kindes angezeigt ${ }^{31}$. Auch beim Entscheid über längerfristige Kindesschutzmassnahmen wie eine Beistandschaft, der durch eine Kindes- und Erwachsenenschutzbehörde gefällt werden muss, ist es für das «Gelingen» der Massnahme und das Erreichen der damit verbundenen Ziele wichtig, dass Eltern und Kinder sich am Verfahren beteiligen können. Die dazu nötigen Abklärungen werden meist von Sozialarbeitenden durchgeführt, Pädiater und Kinder- und Jugendpsychiaterinnen werden jedoch oft für die Abklärung spezifischer Fragestellungen beigezogen. Ein Projekt des Schweizerischen Nationalfonds (SNF) untersucht aktuell, wie sich Eltern und Kindern in solchen Verfahren einbringen können und was sie sich diesbezüglich wünschen ${ }^{32)}$.

Eltern wollen in aller Regel «das Beste» für ihr Kind und dessen Entwicklung. Allerdings können sich elterliche psychische Störungen und Suchterkrankungen temporär oder dauerhaft auf die elterliche Erziehungsund Urteilsfähigkeit auswirken. Die Fähigkeit, das jeweils «Beste» oder für die individuelle Entwicklung eines Kindes Notwendige zu gewährleisten, kann dadurch empfindlich eingeschränkt sein. Zudem werden familiäre Haltungen und Überzeugungen vom soziokulturellen Kontext und den familiären Sozialisationsbedingungen geprägt.

\section{Vereinbarte Zusammenarbeit}

Familien mit einem psychisch oder suchterkrankten Elternteil sind häufig auf mehrfache Weise belastet und nicht selten sind diverse Fachpersonen und Fachstellen involviert. Das kann zur Folge haben, dass viel Zeit aufgewendet werden muss, um sämtliche Informationen der Netzwerkpartner*innen zu erhalten und diese miteinander auszutauschen. Dazu ist eine vereinbarte Zusammenarbeit der Netzwerkpartner*innen auf Quartiers-, Institutions- oder Gemeindeebene anzustreben ${ }^{33)}$. Dies beinhaltet ein transdisziplinäres Denken und impliziert eine verstärkte Perspektivenübernahme und Zusammenarbeit zwischen Disziplinen, Professionen, Organisationen und Teilversorgungssystemen. Die Koordination und Kooperation kann einerseits fallübergreifend durch Vernetzungs- 


\section{Fortbildung}

gremien, Versorgungsplanung und gemeinsame Trägerschaften erfolgen, andererseits fallbezogen durch koordinierte Hilfeplanung und geklärte Fallführung. Nach Möglichkeit ist im Netzwerk eine gemeinsame und geteilte Verantwortung anzustreben ${ }^{34)}$. Die Qualität und Funktionalität eines Netzwerks ist sehr eng mit den personellen und damit auch finanziellen Ressourcen für eine vereinbarte, systematische und fallunabhängige Zusammenarbeit verknüpft. Datenschutz- und Schweigepflichtsbestimmungen können zu einem eingeschränkten Informationsaustauch zwischen den Netzwerkpartnern führen. Hier kann pragmatisch eine Schweigepflichtsentbindung nützlich sein, die beinhaltet, wer von den Eltern resp. den Erziehungsberechtigten ermächtigt wurde, mit welchen Drittpersonen Kontakt aufzunehmen und in welche Richtung der Informationsaustausch gewünscht wird. Zurückliegende Erfahrungen zeigen, dass es ein unablässiges Engagement des Netzwerks und einige «Thementräger» braucht, um eine funktionierende und lebendige integrierte Versorgung aufzubauen und langfristig zu erhalten.

\section{Fazit}

Eine gelingende Unterstützung von Familien mit komplexen Bedürfnissen erfordert eine gemeinsame tragende Grundhaltung und einen engen fachlichen und persönlichen Austausch des Hilfesystems. Für Kinder, Jugendliche und Familien mit einem psychisch oder suchterkrankten Elternteil sind Vertrauen, Kontinuität und Verlässlichkeit wichtige und bestimmende Garanten einer gelingenden Zusammenarbeit mit Fachpersonen. Darüber hinaus ist eine weitere Sensibilisierung von Öffentlichkeit, Fachpersonen, Politik und Wirtschaft erforderlich, damit psychische Gesundheit selbstverständlicher thematisiert und relevante Faktoren des «well-being» in den Alltag integriert werden. Insbesondere ist an Schulen gemeinsam mit Lehr- und Betreuungspersonen, Eltern sowie den Schüler*innen Aufklärungsarbeit für psychische Gesundheit im Sinne von Prävention und Frühintervention zu leisten und das schulinterne und regionale Netzwerk an Fach- und Betreuungspersonen bei Bedarf einzubeziehen.

Für das Literaturverzeichnis verweisen wir auf unsere Online Version des Artikels.

\footnotetext{
Autoren

Dr. med. Kurt Albermann, Chefarzt, Sozialpädiatrisches Zentrum SPZ, Departement Kinder- und Jugendmedizin, Kantonsspital Winterthur Dr. med. Brigitte Müller, Psychologin FSP, Hochschule für Soziale Arbeit FHNW, Institut Kinder- und Jugendhilfe, Muttenz

Die Autoren haben keine finanziellen oder persönlichen Verbindungen im Zusammenhang mit diesem Beitrag deklariert.
} 